\title{
Active learning of introductory optics: real-time physics labs, interactive lecture demonstrations and magic
}

\section{David Sokoloff}

David R. Sokoloff, "Active learning of introductory optics: real-time physics labs, interactive lecture demonstrations and magic," Proc. SPIE 9664, Ninth International Topical Meeting on Education and Training in Optics and Photonics, 96642R (24 October 2005); doi: 10.1117/12.2207768

SPIE Event: Ninth International Topical Meeting on Education and Training in Optics and Photonics, 2005, Marseille, France 


\title{
Ref ETOP085
}

\section{Active Learning of Introductory Optics: RealTime Physics Labs, Interactive Lecture Demonstrations and Magic}

\author{
David R. Sokoloff \\ Department of Physics, 1274 University of Oregon, Eugene, OR 97403-1274 USA \\ sokoloff@darkwing.uoregon.edu
}

\begin{abstract}
Widespread physics education research has shown that most introductory physics students have difficulty learning essential optics concepts-even in the best of traditional courses, and that well-designed active learning approaches can remedy this problem. This mini-workshop and the associated poster session will provide direct experience with methods for promoting students' active involvement in the learning process in lecture and laboratory. Participants will have hands-on experience with activities from RealTime Physics labs and Interactive Lecture Demonstrations-a learning strategy for large (and small) lectures, including specially designed Optics Magic Tricks. The poster will provide more details on these highly effective curricula.
\end{abstract}

\section{Keywords}

Introductory physics, active learning, introductory optics, introductory laboratory, lecture demonstrations, activity based physics, image formation

\section{Summary}

There is considerable evidence that traditional approaches are ineffective in teaching physics concepts, including light and optics concepts. ${ }^{1}$ A major focus of the work at the University of Oregon and at the Center for Science and Mathematics Teaching (CSMT) at Tufts University has been on the development of active, discovery-based curricula like RealTime Physics labs ${ }^{2}$ and Interactive Lecture Demonstrations. ${ }^{3}$ Among the characteristics of these curricula are:

- Use of a learning cycle in which students are challenged to compare predictionsdiscussed with their peers in small groups - to observations of real experiments.

- Construction of students' knowledge from their own hands-on observations. Real observations of the physical world are the authority of knowledge.

- Confronting students with the differences between their observations and their beliefs.

- Observation of results from real experiments in understandable ways -often in real time with the support of microcomputer-based tools.

- Encouragement of collaboration and shared learning with peers.

- Laboratory work is often used to learn basic concepts.

With the use of this learning cycle and the microcomputer-based tools it has been possible to bring about significant changes in the lecture and laboratory learning environments at a large number of universities, colleges and high schools without changing the lecture/laboratory structure of the introductory physics course. RealTime Physics and Interactive Lecture Demonstrations are described briefly below:

RealTime Physics Active Learning Laboratories (RTP): RealTime Physics is series of lab modules for the introductory physics course that often use computer data acquisition tools to help students develop important physics concepts while acquiring vital laboratory skills. Besides data acquisition, computers are used for basic mathematical modeling, data analysis 
and some simulations. RTP labs use the learning cycle of prediction, observation and comparison. They have been demonstrated to enhance student learning of physics concepts. ${ }^{1,4}$ There are four RTP modules, Module 1: Mechanics, Module 2: Heat and Thermodynamics, Module 3: Electric Circuits and Module 4: Light and Optics. ${ }^{2}$ Each lab includes a pre-lab preparation sheet to help students prepare, and a homework, designed to reinforce critical concepts and skills. A complete teachers' guide is available online for each module.

Interactive Lecture Demonstrations (ILDs): ILDs are designed to enhance conceptual learning in large (and small) lectures. Real physics demonstrations are shown to students, who then make predictions about the outcomes on a prediction sheet, and collaborate with fellow students by discussing their predictions in small groups. Students then observe the results of the live demonstration (often displayed as real-time graphs using computer data acquisition tools), compare these results with their predictions, and attempt to explain the observed phenomena. Besides data acquisition, computers are used for interactive video analysis. The eight-step ILD procedure incorporating this learning cycle is followed for each of the basic, single concept demonstrations in an ILD sequence. ILDs have been demonstrated to enhance student learning of physics concepts. ${ }^{1,5}$ Complete materialsincluding student sheets and teachers' guides-are available for most introductory physics topics. ${ }^{3}$

In this workshop, after an introduction to active learning, participants will work hands-on with an activity from RealTime Physics Light and Optics Lab 3, Geometrical Optics-Lenses, designed to teach the concepts of image formation using two miniature light bulbs (point sources) and a cylindrical lens. Then, the eight-step Interactive Lecture Demonstration procedure will be illustrated through active audience participation. Examples will be drawn from the four sets of optics ILDs: Reflection and Refraction of Light, Image Formation with Lenses, Mirrors and Polarized Light. Guidelines for creating effective ILDs will also be discussed.

A series of Optics Magic Tricks that introduce students to basic optics concepts will also be presented. There are a total of 10 tricks, illustrating concepts in reflection and refraction, image formation with mirrors, total internal reflection, light scattering and polarization. ${ }^{6}$ These include The Reappearing Test Tube, an illustration of the importance of index of refraction for seeing transparent media, Carbon to Silver, an example of total internal refraction and Candle Burning Under Water, an illustration of image formation with a plane mirror. All are presented in a highly active way through the use of optics conceptual learning questions.

Results of studies on the effectiveness of these approaches using the Light and Optics Conceptual Evaluation - developed in conjunction with the curricula-will also be presented.

The poster will present examples from the six labs that make up RealTime Physics Light and Optics. These are Lab 1: Introduction to Light, Lab 2: Reflection and Refraction of Light, Lab 3: Geometrical Optics_Lenses, Lab 4: Geometrical Optics-Mirrors, Lab 5: Polarized Light and Lab 6: Waves of Light. The labs will be displayed along with apparatus, computer data acquisition tools and typical experimental results. Copies of the RealTime Physics Light and Optics lab manual and of the Interactive Lecture Demonstrations book will be available.

All of these curricula have been used successfully by the author in his introductory college level physics course, and recently in a series of Active Learning in Optics and Photonics (ALOP) workshops in Africa (Ghana and Tunisia), sponsored by UNESCO, ICTP and SPIE. More details on the ALOP project will be presented in a separate paper, "Active Learning in Optics and Photonics: Experiences in Africa," with principal author M. Alarcon. 


\section{References}

1. Ronald K. Thornton and David R. Sokoloff, "Assessing Student Learning of Newton's Laws: The Force and Motion Conceptual Evaluation and the Evaluation of Active Learning Laboratory and Lecture Curricula," American Journal of Physics 66, 338-352 (1998).

2. David R. Sokoloff, Ronald K. Thornton and Priscilla W. Laws, RealTime Physics Module 1: Mechanics, Module 2: Heat and Thermodynamics, Module 3: Electric Circuits and Module 4: Light and Optics (Hoboken, NJ, John Wiley and Sons, 2004).

3. David R. Sokoloff and Ronald K. Thornton, Interactive Lecture Demonstrations (Hoboken, NJ, John Wiley and Sons, 2004).

4. Thornton, R.K. and Sokoloff, D.R., "RealTime Physics: Active Learning Laboratory," in The Changing Role of the Physics Department in Modern Universities, Proceedings of the International Conference on Undergraduate Physics Education, 1101-1118 (American Institute of Physics, 1997).

5. David R. Sokoloff and Ronald K. Thornton, "Using Interactive Lecture Demonstrations to Create an Active Learning Environment," The Physics Teacher 35: 6, 340 (1997).

6. David R. Sokoloff, "The Magic of Physics; The Physics of Magic," available from the author. Contains all information needed to perform these "tricks." 